\title{
RegionDCF: a Self-Adapting CSMA/Round-Robin Media Access Protocol for WLAN
}

\author{
Alberto Riggi \\ Information Engineering Department \\ University of Pisa, Italy \\ Lungarno Pacinotti, 43 - 56126 Pisa \\ Email: alberto.riggi@gmail.com
}

\author{
Javier Gomez \\ Telecommunications Engineering Department \\ National Autonomous University of Mexico \\ Av. Universidad 3000, Coyoacan, D.F., 04510 \\ Email: javierg@fi-b.unam.mx
}

\begin{abstract}
This paper presents RegionDCF, a self-adapting media access control protocol for WLAN that seamlessly behaves as CSMA or round-robin access techniques taking advantage of the most effective properties of each access method. In contrast to preceding works in this area that focused on enhancements of each protocol, or on a mechanism that switches between them, this paper proposes a single access method able to behave both as a pure contention-based or as round-robin based protocol depending on present traffic conditions. Simulation results show that RegionDCF outperforms standard 802.11.
\end{abstract}

Keywords: RegionDCF, DCF, Round-Robin, 802.11.

\section{INTRODUCTION}

Wi-Fi technology has experienced an enormous spreading and commercial success in recent years; however, there are unsolved performance issues of this technology, particularly in areas where many nodes contend for the channel such as public hot spots. Wi-Fi uses the Distribution Coordination Function (DCF) as the main access method. In order to reduce collisions among contending stations, DCF employs an exponential backoff mechanism (EB) to introduce some time randomness so stations do not transmit at the same time causing a collision. The EB mechanism, on the average, doubles the time interval a node defers transmission of a packet after each failed attempt. This access delay heavily impacts the utilization of the channel and negatively impacts user experience. As a result it becomes necessary to modify 802.11 to reduce contention delays and improve channel utilization in presence of many contending nodes.

The solution proposed by this paper is a self-adapting access method called RegionDCF, which is based on standard 802.11 DCF. On one hand RegionDCF behaves like a pure CSMA access method for few contending stations and, on the other hand, it behaves like a round-robin access method when many stations contend for the channel. The key idea behind RegionDCF is the concept of region, which is a cluster of stations that can communicate to each other at least at the basic rate. Once a member of a region gains access to the channel using standard DCF method, it grants a contentionfree orderly access to the channel to all other region members in a round-robin manner. Consequently, the contention delays associated to gaining channel access, except for the first one, are eliminated resulting in lower contention delays and a better channel utilization. Channel contention is moved from nodes (as in DCF) to regions by using RegionDCF.

There are many works in the literature that target a reduction of contention delays for the 802.11 standard. In particular, the works using a time-slot approach for WLAN similar to RegionDCF can be characterized as pure TDMA methods or hybrid methods that switch between TDMA and other MACs. In [7] the authors describe the protocols and algorithms to implement TDMA in hardware using an Atheros wireless device. The access method presented in this work is a pure TDMA access method. In [8] the authors propose Soft-TDMAC, a software based TDMA for wireless mesh networks that takes advantage of commodity 802.11 hardware under Linux OS disabling CSMA/CA. In [4] the authors propose STDMA, a pure software TDMA access method to support VoIP applications over wireless LANs. The authors of [3] proposed MultiMac, a framework that allows the concurrent operation of many MAC protocols and the transmission of frames from different access methods within the same framework.

\section{REgiONDCF}

As we reviewed in the previous section most related proposals either emulate TDMA on 802.11 hardware or switch among different MAC protocols. The main difference between these works and the proposed protocol is that RegionDCF is one access method that seamlessly behaves as CSMA or roundrobin (RR) depending on traffic conditions. RegionDCF adapts automatically to traffic conditions without the need to switch between protocols or to signal stations there is a change in the access method, thus maintaining the complexity of the access protocol low. CSMA characteristics, however, are preserved in RegionDCF, especially when contending nodes are few. While RegionDCF can be adapted to ad hoc topologies, it is a protocol initially thought for infrastructure-based networks. In fact, it takes advantage of the particular traffic patterns of these networks where it is common that all traffic is directed exclusively to the Access Point.

In RegionDCF, nodes are organized in regions. In order to join a region a node must be able to communicate with other nodes in the region at least at the basic rate. With RegionDCF the channel is still gained using a contention method such as DCF, but this contention is moved from nodes to regions. The 
main goal of a region is to allow contention-free transmission of one packet by all its members after one member gained access through standard DCF. This period is called Region Burst (RB). The RB does not have a predetermined length because it depends on the number of backlogged region members and on the specific length of the packets transmitted by each member of a particular region.

In order to implement the concept of region in 802.11 a new Region header is proposed, which is a modified MAC header that includes a Reservation sub-header (RSH) as the one shown in [5] and [6]. This sub-header is transmitted at basic rate. Fields of the RSH header are shown in Figure 1. Every region has a unique ID (RegionId) in the network whereas a node has an ID (MemberId) relative to each region it belongs to. The Reserved Slots Field has a different meaning depending on the node decoding it: for nodes in the same region it indicates how many transmission opportunities (TOp) are available during the current region burst; for the AP it indicates the number of SIFS intervals it has to wait before sending an ACK; finally, for other nodes outside the region, it indicates the amount of time (in terms of SIFS) the channel must be considered busy.

The algorithm that determines the time each region member can access the channel is a round-robin scheme based on the ascending order of Member Ids. Hence, the first region member to transmit automatically fixes the transmission order of the remaining nodes in the region. When a member of a region wins access through DCF, it sets the Reserved Slot field of the RSH to the number of region members that have not transmitted during the present region burst. Nodes that do not belong to the same region set their NAV to the value: Reserved Slots Field + SIFS + ACKTime (see Figure 2).

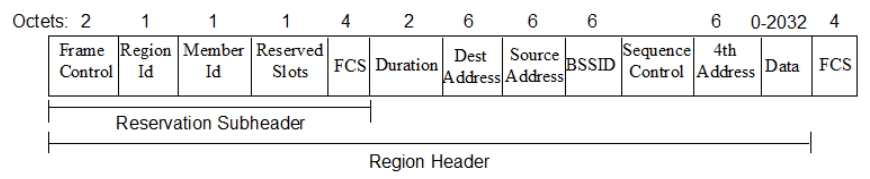

Fig. 1. Region Header

Within a region burst there is a TOp after every SIFS, and all TOps are sorted using a round-robin scheme. Once a node transmits using its corresponding TOp, it sets the Reserved Slots Field to the value of the reserved slots of the previous packet minus the distance calculated between nodes (see Figure 3). Once the region burst finishes the AP sends an aggregated ack to all members of the region after a SIFS period. We call this aggregate ack the Region Ack (RA), shown in Figure 4, where RegionId field is an integer greater than zero. Region ack differs from a legacy 802.11 ack given the presence of the Region Id field that indicates the region where the ack packet is directed. There is also a difference in the way the receiving address (RA) field is used. In 802.11 the RA field is the unicast address to which the packet is destined. In RegionDCF, the RA field is used as a bit mask that indicates which members of the region are acked. The aggregated ack is sent at basic rate so that all nodes of the region can decode

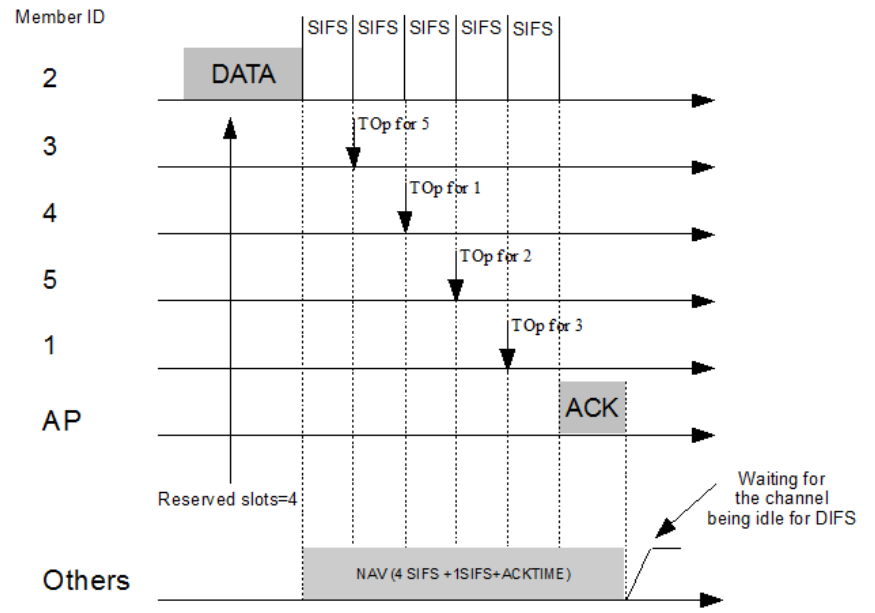

Fig. 2. RegionDCF TOp

the ack packet.

\section{A. Up Link}

Every time a member of a region transmits a packet, the timing of transmission of other nodes in the region is updated in consequence. However, only the start of the TOp for the next member is certainly known (after a SIFS). RegionDCF is based on two timers: the backoff timer and a deferral timer. The former, inherited from DCF, is used to gain access to the channel by the first node that initiated the region burst. Once a member of a region wins the channel, all other region members freeze their backoff timers and start individually a deferral timer by setting this timer to their calculated TOp start value. Members of a region freeze their deferral timer while another region member is using the channel, and reactivate this timer as soon as the channel is sensed idle again. If a transmission from a different region or a collision occurs, the deferral timer is stopped and the backoff timer procedure is reactivated in order to restart contention for the channel.

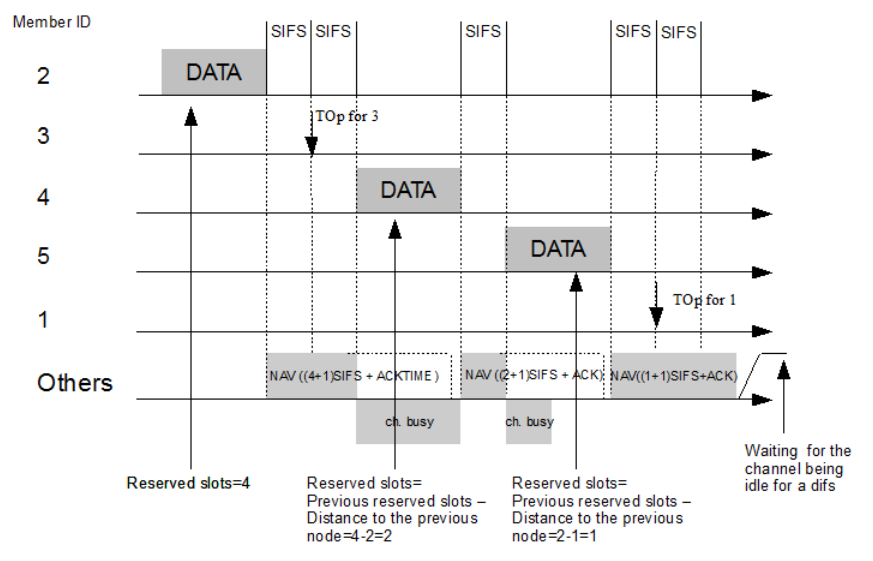

Fig. 3. RegionDCF channel reservation

If a member of a region does not transmit because it has no traffic at the moment, there is only a wasted SIFS interval 
in RegionDCF, which is much shorter than a wasted data slot as in TDMA based protocols. Once a region burst ends, the Access Point must send an aggregated ack to acknowledge proper reception of individual packets during the region burst. After receiving a packet from a region member, the AP sets a deferral timer to a number of SIFS equal to the value of the reserved slots field contained in the RSH plus one (guard) SIFS.

\section{B. Down link}

In the downlink case the AP contends for the channel as any other node in DCF mode in order to transmit its own traffic. Compared to other nodes, the AP has a lower probability to access the channel since it cannot take advantage from other nodes gaining the channel for it. To address this problem, we propose to provide a contention-free period for the AP called AP burst, during which the AP can send a packet back to each member of a region. The chosen region for the AP burst is the one to which the first packet of the AP buffer is directed. The AP keeps sending packets to the selected region as long as the next packet to transmit in its buffer is destined to a member of the chosen region, and that member has not been served already during the current AP burst. Packets in the Access Point buffer, however, are usually not sorted by regions, which may make the AP burst ineffective. In order to alleviate this problem the AP applies a sorting algorithm before the AP burst begins that brings packets destined to members of the chosen region at the head of the AP queue. Only one packet per node in the selected region can be moved up in the queue during the current AP burst. An AP burst can start after an aggregated ack or after the AP gains access to the channel using DCF mode. During the AP burst priority access to the AP is guaranteed because the AP uses a SIFS instead of DIFS to probe the channel is idle.

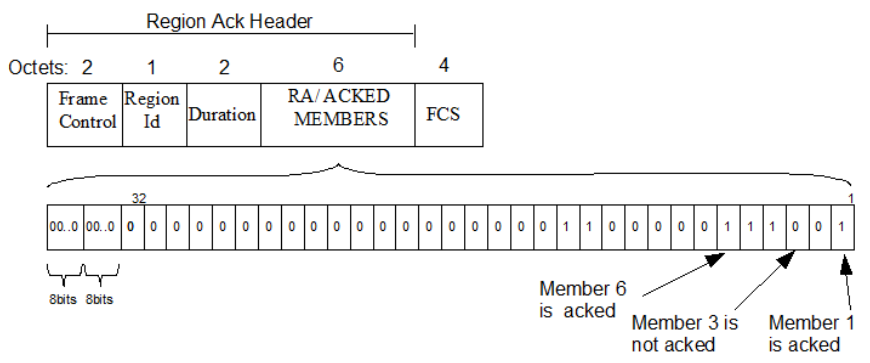

Fig. 4. Region ACK

\section{VALIDATION TESTS}

RegionDCF was implemented in network simulator NS2. We used the ns-miracle extension library [1] to support $802.11 \mathrm{bg}$ rate adaptive functionality. All measured metrics have been evaluated with a 95\% confidence interval being $5 \%$ or $10 \%$ of the mean value through ns-measure [2]. We compare RegionDCF protocol with standard 802.11 bg MAC in terms of throughput, contention time, channel utilization, number of collisions and delay. The tests focused on Frame
Transfer Protocol (FTP). We consider 20 static nodes randomly placed in the network area plus the Access Point located at the center. We considered a network of 200x200 meters to explore what happens when all nodes were within sensing range of each other. Finally, the results presented in this section are expressed in percentage terms comparing the performance of RegionDCF with standard IEEE 802.11bg. The FTP application consisted in the transmission of a file of infinite size so nodes are always backlogged. TCP fragments the file into packets of different size. To make TCP experiments more realistic wireless stations established an FTP connection with a remote node belonging to a wired network connected to the Access Point.

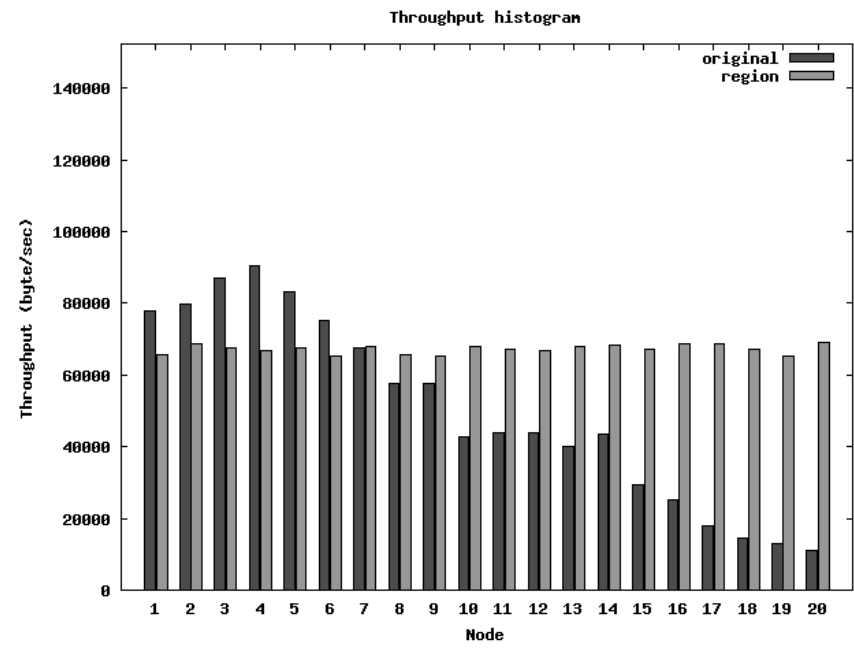

Fig. 5. Individual throughput with regions of five members and FTP traffic

Every wireless station sends FTP traffic to a different node in the wired segment of the network. Each wired node is connected to the AP through a link that has a delay that varies from node to node. In this way both uplink and downlink traffic experiment different delays. In general, results for FTP traffic confirm RegionDCF provides an improvement on network capacity over standard $802.11 \mathrm{bg}$. Figure 5 shows the individual throughput of nodes using standard $802.11 \mathrm{bg}$ and RegionDCF configured with five members per region. The wired links connecting the AP with the wired nodes were configured in a way so that link two has a higher delay than link one, link three has higher delay than link two and so on. The effect of each particular delay can be seen in the individual throughput obtained under standard $802.11 \mathrm{bg}$ in Figure 5. Nodes whose traffic passes through links with higher delays experiment lower throughput. RegionDCF, instead, is more resilient to this problem as it provides similar throughput to all nodes.

Figure 6 shows the aggregated throughput for the three packet sizes being considered while Table I shows a comparison of various performance metrics as we vary the size of regions for the same experiment of Figure 6 but for 512 bytes packets only. As we can observe in Figure 6 and Table 
I, contention times, channel utilization and delays consistently get better for larger regions in Region DCF compared with standard $802.11 \mathrm{bg}$.

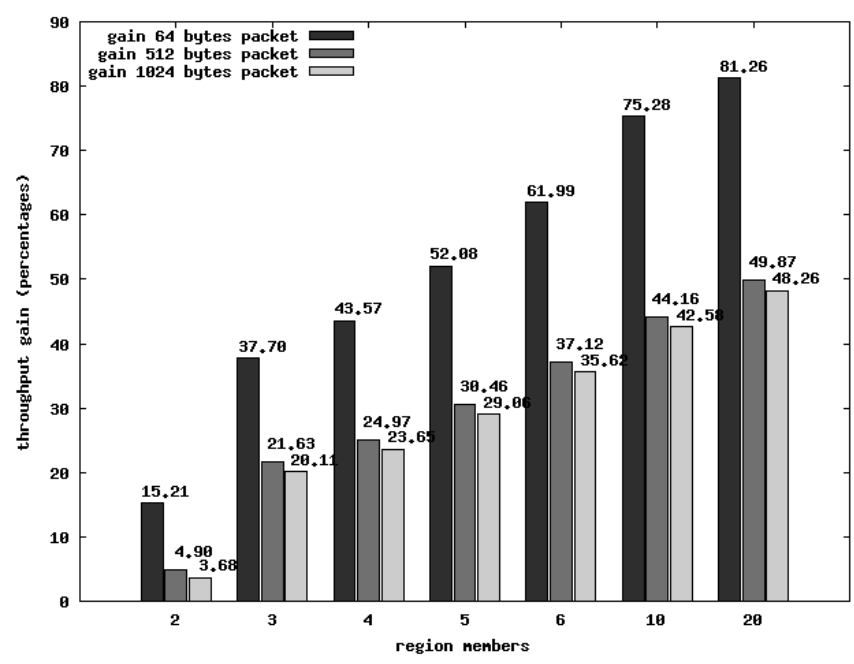

Fig. 6. Wired cum Wireless Scenario, aggregated throughput

TABLE I

COLLECTED METRICS FOR FTP TRAFFIC, P-SIZE 512 BYTES

\begin{tabular}{|l|c|c|c|c|}
\hline $802.11 \mathrm{bg}$ & $\begin{array}{c}2 \text { region } \\
\text { members }\end{array}$ & $\begin{array}{c}5 \text { region } \\
\text { members }\end{array}$ & $\begin{array}{c}\text { 10 region } \\
\text { members }\end{array}$ & $\begin{array}{c}\text { 20 region } \\
\text { members }\end{array}$ \\
\begin{tabular}{|c|c|c|c|c|}
\hline \multicolumn{5}{|c|}{ Contention Time } \\
\hline 0.007362 & 0.005578 & 0.004069 & 0.003012 & 0.001803 \\
& $\mathbf{- 3 2 , 1 8} \%$ & $\mathbf{- 4 4 . 7 3} \%$ & $\mathbf{- 5 9 . 0 9} \%$ & $\mathbf{- 7 5 . 5 1 \%}$ \\
\hline $58.23 \%$ & $61.64 \%$ & $77.84 \%$ & $83.86 \%$ & $86.60 \%$ \\
\hline \multicolumn{5}{|c|}{ Dennel utilization } \\
\hline 0,096906 & 0,058810 & 0.044426 & 0.030665 & 0.035963 \\
& $\mathbf{- 3 3 . 2 2} \%$ & $\mathbf{- 6 4 . 0 3} \%$ & $\mathbf{- 7 5 . 1 7} \%$ & $\mathbf{- 7 0 . 8 9} \%$ \\
\hline
\end{tabular}
\end{tabular}

\section{REGION CONFIGURATION AND MAINTENANCE}

Given that RegionDCF targets wireless networks with infrastructure, the AP is the entity that can better accomplish the task of creating and maintaining regions. The only information that is not available to the AP is the relative distance among nodes. In order to provide this information the following protocol can be implemented. When a node joins the network, it sends a broadcast packet to all neighbors to request information about regions they are currently members of. Nodes that receive the request reply with a small packet containing only the RegionDCF header. These response packets are sent at the basic rate directly to the new node without passing through the AP. In this way the new node can estimate, through signal strength of the received reply packets, the possible achievable rate of each reply packet. The new node sends a packet to the AP that contains a list of all responding nodes and their associated rates. The AP can then estimate the relative location of the new node and assign it to a region. The criteria to assign a new node to a region are:
- Members of a candidate region must all be present in the information packet sent by the new node, or in the database information regarding the new node. In case there are many candidate regions, the AP should prefer regions whose members use higher transmission rates compared with the new node. This policy allows a higher tolerance to variations of channel conditions in order to guarantee the correct decoding of the RSH.

- Maintain the number of region members as uniform as possible in order to avoid unfairness problems. During the experiments we found nodes belonging to larger regions obtained higher throughput than nodes belonging to smaller regions. This behavior is expected as nodes in larger regions have more opportunities to transmit.

\section{CONCLUSION}

This document presents a new access protocol for wireless networks with infrastructure named RegionDCF. RegionDCF is a single access method that seamlessly behaves as CSMA or round-robin depending on present traffic conditions. The proposed access protocol is a feasible alternative to the standard 802.11 MAC protocol since it yields a remarkable performance enhancement compared with CSMA access method. This work opens new possibilities for WLAN networks because it provides shorter contention delays in populated WLANs as well as improving MAC performance for different traffic conditions. RegionDCF can be deployed in wireless access networks having a high node population demanding voice and data services.

Acknowledgements: This work was supported in part by research funds from CONACyT grant 105117, DGAPA-PAPIIT grant IN106609, and Texas A\&M University-CONACyT grant 2010-049.

\section{REFERENCES}

[1] Nicola Baldo, Federico Maguolo, Marco Miozzo, Michele Rossi, and Michele Zorzi. ns2-miracle: A Modular Framework for Multi-Technology and Cross-Layer Support in Network simulator 2. In Proceedings of the 2nd International Conference on Performance Evaluation Methodologies and Tools, 2007.

[2] Claudio Cicconetti, Enzo Mingozzi, and Giovanni Stea. An Integrated Framework for Enabling Effective Data Collection and Statistical Analysis with ns-2. In Proceedings of the 2006 Workshop on NS-2: the IP network simulator, 2006.

[3] Doerr Christian et al. MultiMAC - An Adaptive MAC Framework for Dynamic Radio Networking. In Proceedings of IEEE International Symposium on New Frontiers in Dynamic Spectrum Access Networks, 2005.

[4] Fanglu Guo and Tzi cker Chiueh. Software TDMA for VoIP Applications over IEEE 802.11 Wireless LAN. In Proceedings of IEEE Infocom, 2007.

[5] Zhu Hao and Cao Guohong. rDCF: A Relay-Enabled Medium Access Control Protocol for Wireless Ad Hoc Networks. IEEE Transactions on Mobile Computing, vol. 5, no. 9, 2006.

[6] G. Holland, N. Vaidya, and P. Bahl. A Rate-Adaptive MAC Protocol for Multihop Wireless Networks. In Proceedings of ACM Mobicom, 2001.

[7] Sam Leffler. TDMA for Long Distance Wireless Networks. White Paper, 2009.

[8] Djukic Petar and Mohapatra Prasant. Soft-TDMAC: A Soutwore TDMAbased MAC over Ccommodity 802.11 Hardware. In Proceedings of IEEE Infocom, 2009. 